Espacio y Desarrollo No 33, 2019, pp. 9-27 (ISSN 1016-9148)

https://doi.org/10.18800/espacioydesarrollo.201901.001

\title{
«No hay tiempo conforme». Percepciones sobre el cambio CLIMÁTICO EN COMUNIDADES INDÍGENAS DE LA AMAZONÍA PERUANA
}

\author{
Oscar Espinosa \\ Pontificia Universidad Católica del Perú \\ oespinosa@pucp.edu.pe
}

Fecha de recepción: 24 de octubre de 2018

Fecha de aceptación: 10 de mayo de 2019

\section{Resumen}

En la última década, los efectos del cambio climático han venido afectando a las comunidades indígenas de la Amazonía peruana, especialmente a través de eventos climáticos extremos - como sequías o inundaciones anormales - y mediante un cambio general en las estaciones. Este artículo presenta los resultados principales de un proyecto de investigación que tuvo como uno de sus objetivos el recoger las percepciones de distintas comunidades amazónicas sobre dichos cambios. Este proyecto se desarrolló en dos etapas entre los ańos 2011 y 2016, y trabajó con comunidades pertenecientes a cuatro pueblos indígenas distintos: asháninka, awajún, shipibo-konibo y kukama, ubicadas en las regiones de Junín, San Martín, Ucayali y Loreto, respectivamente.

Palabras clave: Amazonía, cambio climático, asháninka, shipibo-konibo, kukama, awajún

\section{«The Time is Not Right»: Perceptions about Climate Change in Indigenous Commu- nities in the Peruvian Amazon region}

\section{Abstract}

In the last decade, the indigenous communities of the Peruvian Amazon region have been experimenting the effects of climate change, especially extreme climatic events such as abnormal droughts and flooding, as well as other seasonal changes. This article presents some of the main results of a research project about the perception of indigenous communities about climate change. The research was developed in two periods, between 2011 and 2016, and the field-work took place in communities of four different indigenous peoples: Asháninka, Awajún, Shipibo-Konibo and Kukama, communities located in the Peruvian regions of Junín, San Martín, Ucayali y Loreto.

Keywords: Amazonia, Climate change, Ashaninka, Shipibo-Konibo, Kukama, Awajun. 


\section{INTRODUCCIÓn}

El cambio climático es uno de los temas más relevantes que se han venido discutiendo en los últimos ańos en materia ambiental, tanto en el Perú como en el resto del mundo. El estudio de sus causas y consecuencias no solamente ha sido abordado desde distintas disciplinas académicas, sino que también ha estado ligado a la discusión de políticas públicas, tanto en los niveles nacionales como supranacionales. En el caso del Perú, el presidente de la República, Martín Vizcarra, promulgó en abril de 2018 la Ley Marco de Cambio Climático para regular la acción del Estado con relación a este problema. A pesar del interés que despierta el cambio climático en el Perú, las políticas públicas y los estudios sobre este fenómeno en el Perú siguen concentrándose en las zonas altoandinas o en sus efectos sobre los recursos hídricos en la costa debido a que los glaciares tropicales son más sensibles a los cambios de temperatura (Carey, 2010), mientras que el interés por comprender, estudiar y actuar frente al cambio climático en la región amazónica ha pasado a un segundo plano, tanto a nivel de políticas públicas como en la investigación académica. Esto, además, a pesar de que el rol del bosque amazónico es fundamental para el país y otras partes del mundo por ser un importante consumidor de calor, una reserva de carbono y un factor determinante en el 50\% de las lluvias en la zona que normalmente son producidas por la evapotranspiración (Espinosa, Gonzáles y Taller de Amazonía, 2014).

En el caso de la región amazónica peruana, además, en la última década se han producido, de manera muy seguida, eventos climáticos extremos (Fearnside, 2012; Marengo, Borma, Rodriguez, Pinho, Soares y Alves, 2013; Marengo y Espinoza, 2015; Pabón-Caicedo, Ycaza, Friend, Espinoza, Fenzl y Apostolova, 2018). En los años 1998, 2005, 2010 y 2016, se produjeron sequías extremas, y tres de ellas se encuentran entre las peores sequías de los últimos ciento veinte años (Marengo et al., 2008; Marengo, Nobre, Tomasella, Cardoso y Oyama, 2008; Espinoza et al., 2011a; Espinoza et al., 2011b; Espinoza et al., 2012; Lewis, Brando, Phillips, Van der Heijden y Nepstad, 2011). Según Jiménez-Muñoz et al. (2016), el año 2015 fue el más caluroso en la región amazónica desde 1900. La sequía del año 2016, vinculada al conjunto de efectos climáticos asociados al fenómeno de El Niño ha sido considerada como una de las más graves, quizás la peor en cincuenta años, impactando un territorio mayor que otras anteriores (Erfanian, Wang y Fomenko, 2017). Asimismo, se ha comprobado que desde 1980 se está incrementando la duración de la temporada seca (Espinoza, Segura, Ronchail, Drapeau y Gutiérrez-Cori, 2016).

También se han producido fuertes inundaciones en 1999, 2009, 2011, 2012, 2014 y 2015, estas dos últimas principalmente en la Amazonía sur del Perú, así como en las regiones amazónicas de Bolivia y de los Estados de Acre y Rondonia en Brasil (Espinoza, Marengo, Ronchail, Molina, Noriega y Guyot, 2014; Marengo y Espinoza, 
2015). Las inundaciones de 2011 y 2012 han sido consideradas como dos de las peores inundaciones, alcanzando los ríos caudales muy grandes que solamente habían tenido lugar en la región otras dos veces más durante el último siglo. En el mes de abril 2012 se obtuvo el récord histórico de crecimiento de caudal en la Amazonía peruana (Espinoza et al., 2012; Espinoza et al., 2014; Espinoza, Chavez, Ronchail, Junquas, Takahashi y Lavado, 2015; Lavado, Labat, Ronchail, Espinoza y Guyot, 2013).

Uno de los impactos más graves del cambio climático en la región amazónica es el que afecta la biodiversidad y a la alimentación y salud de las poblaciones locales. Las sequías e inundaciones extremas, junto con los cambios en los vientos y en el ritmo de las estaciones ha generado la desaparición de diversas especies de flora y fauna (Espinosa et al., 2014)(citar algunas y las fuentes). Estos cambios modifican las épocas en las que las plantas florecen o fructifican. La alteración en el ciclo anual de las plantas también tiene un impacto directo sobre los animales y los seres humanos que consumen sus frutos. Por ejemplo, según la Fundación Bustamante de la Fuente (2010), en los últimos ańos se han notado alteraciones importantes en el ciclo vital de algunas plantas como el camu camu (Myrciaria dubia), del pijuayo (Bactris gasipaes) o del aguaje (Mauritia flexuosa). Todo esto produce un cambio en los hábitos de alimentación y tiempos de recolección de las poblaciones, lo que, a su vez, genera un desbalance en sus costumbres. Al cambio climático, hay que sumar además la deforestación, la tala y minería ilegal, y la contaminación de los ríos con químicos y con ruido, también vienen impactando negativamente en la biodiversidad y en el acceso de la población a sus recursos naturales.

\section{EL CAMBIO CLIMÁTICO Y LAS POBLACIONES INDÍGENAS EN LA REGIÓN AMAZÓNICA PERUANA}

El cambio climático afecta de manera más radical a las sociedades indígenas, sobre todo a aquellas que se encuentran en una relación de mayor dependencia con el medio ambiente. Como señala el Foro Permanente para las Cuestiones Indígenas de la ONU:

[el] cambio climático constituye una amenaza y un peligro para la supervivencia de los pueblos indígenas en todo el mundo, pese a que los pueblos indígenas contribuyeron muy poco a las emisiones de gases de efecto invernadero. De hecho, los pueblos indígenas son esenciales para los numerosos ecosistemas que habitan sus tierras y territorios y forman parte activa de ellos, por lo que podrían ayudar a mejorar su resiliencia. Además, los pueblos indígenas interpretan los efectos del cambio climático, y reaccionan ante ellos, de manera creativa, aprovechando los conocimientos tradicionales y otras técnicas para encontrar soluciones que puedan ayudar a la sociedad en su conjunto a hacer frente a los cambios inminentes. (United Nations Permanent Forum on Indigenous IssuesUNPFII, 2008, p. 2) 
El trabajo con las comunidades indígenas en relación con el cambio climático resulta fundamental, no solamente en la medida en que son sociedades que dependen fuertemente de las condiciones ambientales para reproducir sus medios de subsistencia (Echeverri, 2009), sino también porque sus conocimientos sobre el medio ambiente, a veces definidos como "etno-ecología», puede aportar elementos indispensables para ayudar a comprender y responder los retos del cambio climático (Ortiz, 2015; Edington, 2017).

En el caso de la región amazónica, las comunidades indígenas que se hallan fuertemente vinculadas a la agricultura, la recolección, la caza y la pesca para la subsistencia son especialmente vulnerables a estos cambios. La frecuencia e intensidad de los eventos extremos y los cambios en las estaciones hacen que sea cada vez más difícil predecir los efectos del clima sobre su entorno, generando problemas para conseguir alimentos, una mayor exposición a enfermedades, dificultades para acceder a agua potable, pérdida de bienes, entre otras. Pese a ello, lejos de asumir una actitud pasiva, los pueblos amazónicos han desarrollado estrategias de adaptación a los cambios del medio ambiente. Estas adaptaciones han significado cambios en las rutinas diarias, trabajo, patrones de consumo y prácticas culturales de las sociedades amazónicas (Echeverri ,2009). Además, en la medida en que las comunidades manejen bioindicadores (en el caso de animales o plantas) o de hidroindicadores (las variaciones en los niveles de los cuerpos hídricos, en las precipitaciones), mayores serán las posibilidades de hacerle frente a un fenómeno climático que, de otra manera, resultaría en desastre.

En términos de investigación, sin embargo, los estudios dedicados a las percepciones, saberes y estrategias de las comunidades indígenas siguen siendo escasos. Para el caso de la Amazonía peruana, algunos de estos tienen un carácter introductorio y están orientados a sensibilizar a la población en general o a la población indígena sobre el tema (Müller, 2009; Proyecto Andino de Tecnologías Campesinas-PRATEC, 2009). Entre los trabajos focalizados en comunidades indígenas amazónicas en el Perú, se encuentran las tesis de Sidney Novoa (2013) y Lucía Perea (2015) con comunidades sharanahua y cashinahua, respectivamente, en la zona del río Purús; el estudio de Chocano (2010) en la comunidad awajún de Nazareth; la tesis de Tuesta (2014) en tres comunidades indígenas cercanas a Iquitos (una kukama, una yagua y una bora); el de Dan Rosengren (2016) con comunidades matsigenka del río Urubamba, y el ya mencionado trabajo de Espinosa et al. (2014) en una comunidad shipibo-konibo del Ucayali y tres comunidades awajún en la región San Martín. A estas investigaciones se podría añadir la de la Sociedad Peruana de Derecho Ambiental (SPDA) con población ribereña de la comunidad campesina El Chino, en la quebrada de Tamshiyacu, en Loreto (Ruiz, Müller y Flórez, 2012a), y la tesis de Joel Lozano (2016) en la zona periférica de la ciudad de Iquitos. Finalmente, habría que mencionar también el 
proyecto piloto sobre salud y cambio climático en comunidades indígenas amazónicas en el Perú, implementado por la Universidad Cayetano Heredia como parte de una investigación internacional sobre adaptación y respuestas locales al cambio climático (Hofmeijer, Ford y Berrang-Ford, 2012).

A estas investigaciones realizadas en la Amazonía peruana, habría que añadir aquellas realizadas en otras comunidades indígenas de la cuenca amazónica en su conjunto, sobre todo aquellas hechas sobre Colombia (Remuy, 2008; Ulloa, 2008; Echeverri, 2009 y 2010; Kronik y Verner, 2010; Ruiz, Müller y Flórez, 2012b, Henao y Farekatde, 2013), Brasil (Faulhaber, 2004; Cardoso, 2008; Silva, Cardoso, Fonte y Rodrigues, 2010; Coordenação das Organizaçôes Indígenas da Amazônia BrasileiraCOIAB y Centro Amazônico de Formação Indígena-CAFI, 2011; Oliveira, Mafra y Soares, 2012; Ramos, 2012) y Bolivia (Padraza y Pachaguaya, 2010; Ferreira Arza, 2011; Fernández-Llamazares et al., 2014), a los que se puede sumar también aquellos realizados en la región de la Chiquitanía boliviana (Gonzáles, 2012; Ruiz, Müller y Flórez, 2012c).

Los resultados que se presentan a continuación corresponden a un proyecto de investigación en dos etapas que comenzó en el año 2011 y terminó a fines del año 2016. En la primera etapa, se realizó el trabajo de campo entre los ańos 2011 y 2012 en tres comunidades ubicadas en la selva alta y una en la selva baja. Las comunidades en la selva alta fueron las comunidades awajún de Shampuyacu, Alto Naranjillo y Bajo Naranjillo en la región San Martín, mientras que la comunidad de selva baja fue la comunidad shipibo-konibo de Nuevo Saposoa en la región Ucayali. En la segunda etapa, que se implementó entre los años 2013 y 2016, se realizó el trabajo de campo entre los años 2015 y 2016 en tres comunidades de selva baja y tres comunidades de selva alta. Las comunidades de selva baja fueron las comunidades kukama de Atenas, Shapajilla y Cuninico sobre el río Marañón en la región de Loreto, mientras que las de selva alta fueron las comunidades asháninka de Potsoteni, Alto Camonashiari y Cheni, ubicadas en el distrito de Río Tambo, región Junín.

Se decidió trabajar de manera explícita en regiones de «selva alta» (piedemonte andino-amazónico) y de «selva baja» o llanura amazónica debido a que los impactos del cambio climático son diferentes en cada una de estas regiones. En la «selva alta» son más fuertes las sequías, llegándose incluso a presentar casos de incendios forestales a pesar de ser bosques tropicales húmedos; asimismo el impacto por la deforestación es más grande; y, en época de lluvias, pueden presentarse derrumbes y aluviones. En el caso de la selva baja, por el contrario, los eventos extremos, tanto sequías como inundaciones, impactan de manera más fuerte en los niveles de los ríos, afectando así las actividades económicas y la vida cotidiana de la gente que vive cerca de estos. 


\section{LA PERCEPCIÓN DE LOS CAMBIOS CLIMÁTICOS EN LAS COMUNIDADES DE SELVA BAJA}

\section{a) El caso de la comunidad shipibo-konibo de Ucayali}

En la comunidad de Nuevo Saposoa existe una noción general de que el clima está cambiando. Esta percepción se identificó a partir de dos fuentes de información. Una de ellas es la experiencia misma de la gente en relación con su entorno, mientras que la otra se debe a la información provista por la escuela o por distintas instituciones que han llegado a la comunidad para trabajar el tema del cambio climático.

En el caso de los y las jóvenes, este último medio ha sido muy importante, y en muchos casos manejan más información sobre el cambio climático en el mundo que los impactos de este en la comunidad. Por el contrario, la población adulta, y sobre todo los adultos mayores, son quienes tienen una percepción más detallada y precisa sobre los impactos del cambio climático en su entorno debido a que están interactuando permanentemente con este como parte de sus actividades cotidianas como, por ejemplo, en la pesca, que es una de las principales actividades de la comunidad. Así, por ejemplo, muchas personas de la comunidad señalaron que en los últimos años los peces grandes son escasos, y que luego de las grandes inundaciones recientes hay menos peces, especialmente el paiche (Arapaima gigas) y la gamitana (Colossoma macropomum). Resulta interesante notar que las personas de la comunidad relacionan este fenómeno con el cambio climático y no, por ejemplo, con la pesca, contaminación u otros factores.

Las inundaciones extremas también han afectado a la agricultura porque se ha perdido una parte importante de los barrizales, que son las zonas que reciben los nutrientes que arrastra el río y que se pueden sembrar durante la época de verano ${ }^{1}$, cuando desciende el nivel de los ríos, al destruir varias veces los cultivos. La imprevisibilidad o la intensidad de estas, dificulta planificar esta actividad. Y, además, debido al incremento de la temperatura, las playas que se forman al descender el caudal no cuentan con la suficiente humedad o nutrientes que requieren los cultivos que allí se siembran. Asimismo, cuando se han producido inundaciones extremas, el caudal del río ha tardado en bajar hasta dos meses más de lo usual, postergando el tiempo en que termina de secar lo suficiente para poder sembrar. Así, estas chacras, en vez de haber sido sembradas a partir de abril, recién se comenzaron a sembrar en agosto. Este atraso implica entonces, tener que cambiar los productos agrícolas, buscando cultivos de menor duración como, por ejemplo, variedades de yuca que puedan cosecharse en tres o cuatro meses, y no las variedades que usualmente se consumen y que demoran nueve meses.

\footnotetext{
1 En la región amazónica peruana se denomina «verano» a la temporada seca o de vaciante de los ríos, mientras que el «invierno» corresponde a la temporada de lluvias o de creciente de los ríos. Estas temporadas varían de un lugar a otro y no corresponden necesariamente a las estaciones de verano o de invierno propias del hemisferio sur.
} 
A pesar de que cada vez son más frecuentes este tipo de situaciones de imprevisibilidad y de vulnerabilidad, la mayoría de la comunidad considera que estos eventos no son tan graves, y más bien tratan de ubicar los eventos de sequía e inundación extremos como parte de un fenómeno cíclico que se repetiría cada veinte ańos, aproximadamente. Y de manera consecuente, buscan soluciones concretas y pragmáticas inmediatas, antes que tratar de encontrar una explicación más general a los cambios ambientales que se están produciendo en el mediano y largo plazo. Una de estas soluciones ha sido el acuerdo de la asamblea comunal de construir las viviendas en zonas más elevadas para evitar que las inundaciones extremas impacten de manera directa sobre la salud o la vida cotidiana de las familias. Sin embargo, algunas familias también han optado por migrar a otras partes, ya sea a lugares más alejados del río, o bien incluso, hacia ciudades como Pucallpa, que es la capital de la región.

\section{b) El caso de las comunidades kukama del bajo Marañón, Loreto}

En el caso de las comunidades kukama con las que se trabajó, la población es más consciente de los impactos del clima sobre los niveles del río Marañón. Y al igual que en el caso de la comunidad de Nuevo Saposoa, ha crecido la sensación de incertidumbre y de imprevisibilidad sobre las estaciones y el momento en que el descenso del río les puede permitir sembrar en las playas o barrizales.

La variación en las estaciones no permite a la gente seguir el ritmo acostumbrado para sembrar o cosechar. Como señalaba un comunero de Cuninico: «ahorita no tenemos verano, sigue lloviendo y ya estamos en el mes de julio, no hay verano, pero anteriormente el mes de julio era pleno verano aquí [...] pero ahora no se ve el verano, o sea que no podemos decirle exactamente cuándo, a qué fecha» (Comunero de Cuninico, comunicación personal, 2016).

Con la temporada de invierno se da una situación similar, ya que los meses de lluvia van cambiando cada ańo, y no es posible prever el tiempo. Por ejemplo, en el ańo 2015 el invierno duró hasta junio, mientras que en 2016 empezó tarde, en marzo, y terminó temprano, en mayo. Incluso algunas personas mencionaron que ya no se podía hablar de verano o invierno, porque todo estaba variando. Algunos años las lluvias comienzan en diciembre, otros en febrero, otros en marzo; y de manera similar, a veces terminan en febrero y otros años en mayo o junio. A veces dura poco tiempo, como en 2015, que duró solo dos meses, pero otros años dura seis meses. El señor César Mozombite señala: "ahora ya no hay verano conforme, no hay invierno conforme» (Comunicación personal, 2015); y otro comunero ańade: «no sabemos si el invierno se va o si va seguir todavía», mientras que uno tercero concluye: «ha cambiado el clima totalmente» (Comunero de Cuninico, comunicación personal, 2015). 
El principal impacto de esta incertidumbre sobre el clima se da en relación con la agricultura. Como señala una señora kukama:

Antes tenía su época de verano, y el invierno, pero ahora ya no. Estamos mirando en estos tiempos que todos los días, pasa un día de veranito, dos días de veranito, viene la lluvia, se puede decir en la mayoría estamos mirando el invierno. Antes no, antes teníamos por decir hacíamos nuestra chacra en el mes de, por decir ahorita estamos en julio ¿di? Julio era el verano ya totalmente, pero ahorita no. Julio, agosto, septiembre, octubre [era verano]; noviembre ya era el invierno, pero ahora casi todos los días. En este tiempo ya la taricaya [Podocnemis unifilis] veíamos en las playas grandes y esos animales salían ya a poner sus huevitos. Pero ahora no se ven esos animales. No habrá verano conforme, porque no hay playa conforme ahora, y eso quiere decir que el tiempo ha cambiado, ya no es el tiempo de antes (Comunera de Cuninico, comunicación personal, 2016).

Asimismo, hay una mayor percepción sobre el incremento de la temperatura. Antiguamente, la gente trabajaba en sus chacras de manera corrida, pero el incremento de calor hace que muchas familias opten por descansar o volver a sus casas entre las 10 am y las 4pm en que "quema tanto sol». Este incremento en la temperatura también es percibido como la causa de que muchas plantas se marchiten (como los mashquis o hijuelos de los plátanos) o que las semillas no broten.

También se ha acentuado el frío y los vientos. En esta región, hacia fines de junio suele darse un pequeño período que dura entre tres y siete días en que la temperatura baja, y este tiempo es popularmente conocido como «el frío de San Juan» debido a la fiesta de este santo que se celebra el 24 de junio; mientras que, hacia fines de agosto, se suele producir otro período de frío acompañado de fuertes vientos. A estos días de viento se les conoce como «los vientos de Santa Rosa», ya que la fiesta de esta santa tiene lugar el 30 de agosto. Sin embargo, en años recientes estos períodos de frío y viento llegan en otros momentos, antes o después de las fechas previstas, y se han estado presentando, además, en algunos años de manera más intensa, o en otros años casi desapercibidos.

Finalmente, es importante mencionar que, para las comunidades kukama, relacionan los cambios climáticos, propiamente dichos, con otros cambios ambientales, como la contaminación de los ríos, que están impactando sobre su vida y su salud, y también sobre la vida de los animales, tanto peces como aves y mamíferos.

\section{LA PERCEPCIÓN DE LOS CAMBIOS CLIMÁTICOS EN LAS COMUNIDADES DE SELVA ALTA}

\section{a) El caso de las comunidades awajún del Alto Mayo, San Martín}

En el caso de las comunidades awajún, la población identifica diversos cambios en el medio ambiente. En primer lugar, muchas personas señalaron que antes el clima era más fresco, ya que había más árboles en el bosque («monte»o «montaña»), pero 
que la mayor parte ha sido deforestada. Incluso, en la comunidad de Bajo Naranjillo habían reservado algo más de mil hectáreas como un área de conservación, pero un gran porcentaje de esta zona ha terminado siendo deforestada para hacer chacras para las familias más jóvenes. En este sentido, las comunidades awajún no son ajenas a la dinámica predominante en la región San Martín, que es la zona de la Amazonía peruana que ha sido más deforestada.

En segundo lugar, la gente también percibe un cambio en las estaciones y las temporadas de lluvia. Antiguamente, se sabía cuándo llegaba el verano a través de la observación de la floración de ciertas plantas, como la «flor blanca» (Acacia polyphilla), o las flores de la topa o tupa (Ochroma lagopus) o de la tangarana (Triplaris sp.). Durante el verano, además de abrir las chacras y sembrar, se salía al monte a cazar animales como el venado gris (Mazama gouzobuira), la perdiz (Tinamus major) o el majaz (Agouti paca), pero que ahora son más escasos. Dentro de la época de verano también se da la temporada de los vientos fuertes, usualmente entre agosto y setiembre, y que marcan el inicio del fin de esta época, y por lo tanto, es el tiempo en que se comienza a cosechar o a recolectar diversos alimentos en el bosque.

El problema ahora es que las estaciones han cambiado. Según los pobladores de estas comunidades awajún, el ciclo de lluvias se ha invertido. Antiguamente, el invierno llegaba a más tardar en enero, e incluso las primeras lluvias ya se habían producido antes, a fines de octubre o noviembre. Pero ahora, llueve muy poco durante estos meses, y por el contrario, ha comenzado a llover también en los meses que van de julio a setiembre, cosa que no ocurría antes. Estas lluvias fuera de la época tradicional afectan sobre todo a las chacras de café y maíz. Es más, para algunos comuneros, la lluvia en estos meses de mitad de año estaría reemplazando la temporada de los vientos fuertes, que ahora se dan de manera muy esporádica. En general, la intensidad de las lluvias se ha reducido, y esto explicaría también la ausencia de vegetación y la desaparición de las cochas o lagunitas que se formaban antes durante la época de invierno.

Otro cambio importante se debe a la deforestación, que no solamente ha reducido el tamaño de los bosques en las partes altas, sino que su reducción ha conllevado la disminución del caudal de los ríos, de tal manera que el agua potable se ha vuelto más escasa, generando problemas para la alimentación, la salud y el uso doméstico. También han desaparecido algunos ambientes naturales originarios, como las collpas, que son zonas ricas en arcilla y sales minerales donde viven y se alimentan diversas especies de mamíferos y de aves, como loros, por ejemplo; así como la desaparición de las cataratas, consideradas por los awajún como zonas sagradas.

La reducción de los bosques ha llevado también a una disminución considerable en la flora y fauna, hasta casi desaparecer especies enteras de árboles o de animales. Entre las especies de árboles que la gente de las comunidades considera que han ido 
desapareciendo se encuentran: el cedro (Cedrela odorata), la caoba (Swietenia macrophylla), el tornillo (Cedrelinga catenaeformis), el mashonaste (Clarisia racemosa), la moena (Nectandra sp.), el bambú o caña de Guayaquil (Bambusoideae sp.), la uvilla (Pourouma cecropiaefolia), el árbol de pan (Artocopus altilis) y diversas especies de palmeras, como la yarina (Phytelephas macrocarpa) cuyas hojas eran usadas para techar las casas, o palmeras que dan frutos como el aguaje (Mauritia flexuosa) o el aguajillo (Mauritia carana), entre otras. Asimismo, la reducción del caudal en los ríos, junto con la contaminación de estos y con una extracción depredadora, ha llevado a que también desaparezcan diversas especies de peces. Prácticamente solo existen las siguientes especies: boquichico (Prochilodus nigricans), carachama (Panaque schaeferi), bagre (Pimelodus sp.), zúngaro (Pseudoplatystoma fasciatum), cahuara (Pterodoras granulosus) y tilapia (Oreochromis niloticus) $)^{2}$.

También se ha producido un deterioro en los suelos, y varios de los productos que cultivan, como la yuca, la sachapapa (Dioscorea trifida), la pituca (Colocasia esculenta), el maíz (Zea mays) o el kión (Zingiber officinale), ya no rinden como antes. Algunos mencionan que es precisamente el uso de productos químicos para abonar o fumigar lo que estaría causando que la yuca se malogre y le salgan gusanos.

Hace pocas décadas no tenían estos problemas. Como señalan varios comuneros, «tenían todo a la mano», porque la naturaleza se lo daba. Esto les posibilitaba, además, gastar menos dinero, ya que se alimentaban y curaban con lo que encontraban en el bosque y en los ríos. Según el jefe o apu de la comunidad de Shampuyacu, hasta hace relativamente poco, las personas no cultivaban tanto y mantenían el monte, que ahora se ha reducido a aproximadamente el $60 \%$ de lo que era antes (Tomás Wajajai, comunicación personal, 2012).

Ante los cambios señalados se está intentando tomar ciertas medidas para preservar el medio ambiente. Un caso particularmente interesante es el de la comunidad de Shampuyacu, que ha decidido destinar 1500 hectáreas para bosques protegidos. Para ello han contratado a tres guardaparques indígenas, uno por cada anexo y uno por la comunidad central. Asimismo, se ha prohibido la pesca con dinamita, así como el uso de químicos en los tres ríos de la zona. Además, se ha limitado la cantidad de pesca que cada persona de la comunidad puede extraer.

\section{b) El caso de las comunidades asháninkas de los ríos Ene y Tambo, Junín}

$\mathrm{Al}$ igual que en los casos de las otras comunidades, las comunidades asháninkas también perciben cambios importantes con relación a las estaciones. Antes, la época

\footnotetext{
2 Es importante mencionar que la tilapia es una especie no originaria de la región amazónica, que fue introducida hace un par de décadas para su cultivo en piscigranjas, y que resultó depredando a otras especies nativas (Bittencourt, Leite Silva, Abdon Silva y Dias, 2014; Padial et al., 2017).
} 
de verano se iniciaba entre abril y mayo y se prolongaba hasta octubre, mientras que el invierno comenzaba en noviembre y terminaba en marzo, teniendo a los meses de enero a marzo como aquellos en que se producían lluvias más fuertes. Incluso se pueden apreciar impactos distintos según la ubicación de las comunidades. Así, por ejemplo, Potsoteni, ubicada en la parte baja del río Ene ha sufrido más las sequías, mientras que Alto Camonashari, a la que se llega por carretera desde Mazamari, ha sufrido lluvias torrenciales que han destruido la carretera.

La apreciación general, en todas las comunidades con las que se trabajó, es que llueve menos en la época de invierno y solea más. Por ello, según algunos comuneros, muchas plantas no crecen adecuadamente o terminan por amarillarse y marchitar. En algunos ańos, como en 2014, por ejemplo, prácticamente no hubo invierno. Y en algunas zonas, como en el bajo Ene, cerca de la comunidad de Potsoteni, se han producido también incendios forestales debido a la excesiva sequedad de las plantas por la falta de lluvias. En otras comunidades, como en Unión Puerto Asháninka y Alto Camonashari recuerdan que el año 2001 se secaron todos los riachuelos que bajan por las quebradas. Según Javier Gamboa, presidente de la Asociación de Padres de Familia (Apafa) de Unión Puerto Asháninka, antes el «clima es todo lluvia; era acá, bien húmedo, húmedo, húmedo. Acá había bastante árbol, húmedo y cantidad lluvia, bastante frío. Ahora ya vemos que ya está cambiado. Ya no hay mucha lluvia y también, me parece, mucho sol» (Comunicación personal, 2015).

En la comunidad de Cheni, ubicada sobre el río Tambo, recuerdan a un «ventarrón» anómalo que tuvo lugar durante el invierno de 2015. Según el jefe de la comunidad, este viento fue tan fuerte que produjo el derribamiento de árboles tanto en el monte, como en las chacras de altura; y aunque no logró derrumbar las plantaciones de cacao y yuca, si las inclinó hacia un lado (Cirilo Domingo, comunicación personal, 2016). Asimismo, estos vientos sacaron los techos de varias casas de la comunidad. Y aunque no hubo muertos ni heridos, este fenómeno quedó grabado en la memoria de la comunidad, ya que «nunca antes» había ocurrido algo similar. Las explicaciones sobre esta anomalía, sin embargo, varían. Para algunos, la causa de este ventarrón se debe a la tala de árboles por parte de una empresa maderera en la comunidad vecina de Poyeni, que habría permitido que los vientos pasen directamente sin ningún tipo de barrera que interrumpa su paso, por lo que han adquirido mayor potencia y velocidad. Otros comuneros consideran que estos vientos fueron enviados por los espíritus protectores de los animales como castigo a los pobladores por sus malas prácticas de cacería.

Otro fenómeno anómalo experimentado en Cheni es lo que la comunidad ha llamado «agua sucia». En el año 2008, debido a las fuertes lluvias, el caudal de la quebrada Cheni, que es un afluente del río Tambo, creció de manera inusual, arrastrando lodo, ramas y animales muertos. Por eso se le llamó «agua sucia», ya que además 
de contener mucho lodo, traía consigo un olor desagradable debido a los peces y animales muertos que arrastró consigo. Al igual que el ventarrón de 2015, «nunca antes» había ocurrido algo así, y tampoco ha vuelto a repetirse en los últimos años.

En la comunidad de Cheni también existe preocupación por la disminución de las lluvias. Según los comuneros más ancianos, antiguamente, cada cinco años, aproximadamente, subía la creciente del río Tambo a niveles mayores a los normales. Sin embargo, en los últimos ańos esto no ha vuelto a ocurrir, y se teme que si esta creciente demora mucho en llegar podría terminar siendo más intensa, y por lo tanto, más peligrosa para la comunidad.

Asimismo, en Cheni coinciden que la estación que más ha variado en los últimos años es el verano. Un tema de conversación constante es que los veranos son cada vez más intensos y calurosos. Este incremento del calor ha afectado los horarios de trabajo en las chacras. Ahora tienen que salir más temprano en la mañana, y luego se ven obligados a interrumpir el trabajo hacia las 10 a.m. debido al intenso calor, mientras que antes recién quemaba fuerte el sol hacia el mediodía.

Algunos años también perciben que el verano tarda en llegar, por lo que no sabían exactamente cuándo comenzar a abrir las chacras y sembrar. Y al mismo tiempo, este atraso afectaba la crianza de animales, pues en la época de invierno se enferman más frecuentemente, sobre todo las crías. O las gallinas no pueden terminar de empollar los huevos en los plazos acostumbrados.

Los cambios en las estaciones también ha estado afectando la recolección de insectos comestibles, como el kentori o chicharra (Cicadidae sp.), cuya recolección se da en los veranos, o el suri $^{3}$ y otros gusanos que se recogen en el monte. Así, por ejemplo, en la comunidad de Cheni, en el año 2015, recién se escuchó por primera vez el sonido de la chicharra hacia fines del mes de junio, cuando en otros años ya se les encuentra desde abril.

En algunos casos, la gente explica estos cambios climáticos por causas del crecimiento de las ciudades o la llegada de las empresas madereras o petroleras. En otros casos, se recurre a relatos más tradicionales, y en otros, finalmente, están relacionados con explicaciones basados en sus creencias religiosas. Así, por ejemplo, la señora Leonor Camacho o la señora Juana Antúnez, ambas comuneras de Cheni, señalan que, así como Dios mandó el diluvio en los tiempos de Noé por el pecado de los seres humanos, ahora llegan otros males, porque Dios le prometió a Noé que no volvería a mandar otro diluvio. Pero, como la gente sigue pecando, "por eso ya se nos vienen todos los males, porque ya no se cree en Dios, inclusive los propios creyentes también son pecadores» (Juana Antúnez, comunicación personal, 2016). La señora

\footnotetext{
3 El suri o larva de palmera a veces es clasificado como Coleopterus sp. Sin embargo, a veces se utiliza el término suri como genérico para los gusanos comestibles.
} 
Leonor recuerda que, cuando era joven, llegó a la comunidad un grupo de misioneros evangélicos que decían que los peces se iban a acabar, los ríos se iban a secar, y ya no iba a haber más animales (Leonor Camacho, comunicación personal, 2016). En ese entonces ella no creía, pensaba que esas cosas eran exageraciones, que no iban a pasar. También decían que los choris (los andinos) iban a entrar a las comunidades y les iban a quitar sus tierras e iba a haber guerra. Ella pensaba «¡cómo nos vamos a matar?, ¿̨cómo va a desaparecer los animales?». Pero luego empezó a pensar que estos misioneros tenían razón, que efectivamente llegaron los choris, y luego Sendero Luminoso, y luego están despareciendo los animales. Por ello, concluye: «todo está en la Biblia, ahí dice que todas esas cosas van a pasar; ya no habrá diluvio como antes, sino que se va a sufrir aquí en la tierra» (Leonor Camacho, comunicación personal, 2016).

\section{Conclusiones}

El principal impacto del cambio climático, para las comunidades con las que se ha trabajado, reside en los cambios que se producen en las estaciones. Es decir, la alteración en los tiempos en que aparece o termina el invierno (época de lluvia) o el verano (época seca) constituye el principal problema para las comunidades. De ahí, expresiones como «no hay tiempo conforme»: las estaciones no corresponden a los tiempos que tenían antes. Estas alteraciones y su imprevisibilidad impiden que las comunidades puedan desarrollar sus actividades económicas — como la siembra, la cosecha o la recolección de frutos en el bosque- de manera normal.

En relación con los eventos extremos de sequía e inundación vividos en los últimos ańos, las comunidades han, obviamente, sufrido su impacto. Sin embargo, en la mayoría de los casos tienden a relativizar su importancia en el largo plazo, al tratar de ubicar estos fenómenos como parte de fenómenos cíclicos. A pesar de ello, la frecuencia con que se dan estos fenómenos está llevando a algunas personas a comenzar a preocuparse al respecto.

Un aspecto importante por resaltar es que no todas las personas de las comunidades comparten la misma percepción del cambio climático. Generalmente, las personas mayores, y en general las personas adultas que se dedican a las distintas actividades económicas relacionadas con el medio ambiente como la pesca o la agricultura son quienes tienen una percepción más fina y realista de lo que viene ocurriendo. Por otro lado, la gente más joven y los adolescentes, cuyo conocimiento sobre el cambio climático proviene, en muchos casos, de lo aprendido en la escuela o en talleres organizados por distintas instituciones que vienen de fuera, tiene una percepción que difiere de la generación anterior.

Los resultados de esta investigación también ponen en evidencia la necesidad de continuar estudiando las percepciones, saberes y estrategias de las comunidades 
indígenas amazónicas en relación con el cambio climático. En la Amazonía peruana existen diversas sociedades indígenas que viven en distintas regiones y que interactúan con diferentes ecosistemas, y sería importante contar con mayor información al respecto. Asimismo, es necesario realizar trabajos que crucen la información proporcionada por las comunidades indígenas con aquella generada por las ciencias ambientales y la meteorología.

Una tarea urgente radica, también, en revisar las políticas de adaptación y mitigación del cambio climático para la Amazonía. Particularmente urgente es tomar medidas respecto a la deforestación de los bosques amazónicos, constituye un aspecto crítico del cambio climático en la región (Malhi y Phillips, 2004; Moutinho y Schwartzman, 2005, Gullison et al., 2007). Esta deforestación representa la principal contribución negativa del Perú al cambio climático, llegando al 47\% (Fundación Bustamante de la Fuente, 2010). La deforestación, además, genera otros efectos en la región misma, como la reducción de lluvias.

Finalmente, resulta imprescindible reiterar la necesidad de ubicar estos estudios en una discusión mayor sobre el rol de las comunidades indígenas en relación con el cuidado del medio ambiente (Espinosa et al., 2014). Si bien es cierto que las sociedades indígenas han desarrollado a lo largo de siglos una serie de estrategias y comportamientos amigables con su medio ambiente, en las últimas décadas enfrentan presiones muy fuertes a partir de la presencia de grandes empresas extractivas (de hidrocarburos, de minería, de madera, etc.), de la agroindustria y de grandes proyectos estatales. No es posible, pues, exigir al indígena que cuide el medio ambiente, mientras que, por otro lado, el Estado y las empresas lo destruyen de manera acelerada. Por ello, cualquier discusión sobre la relación entre los pueblos indígenas y el medio ambiente debe incluir el reconocimiento de la autonomía indígena y de sus propios modelos de desarrollo que cuestionan el modelo económico y de desarrollo prevalente en la actualidad.

\section{Agradecimientos}

En este proyecto interdisciplinario participaron, en distintos momentos, las antropólogas Claudia Grados, Dafne Lastra, Meredith Castro, Roxana Gastelú, Flor Correa, Judith Hernández, Gabriela Ho, Haydée Velasque, Eduardo Pacheco y Guillermo Peláez; las geógrafas Gabriela Gonzáles y Tania Herrera, el ingeniero Víctor Ramos, la bióloga Blanca Álvarez y la psicóloga social Laura Jurado. 


\section{REFERENCIAS}

Bittencourt, L., Leite Silva, U., Abdon Silva, L., Dias, M. (2014). Impact of the invasion from Nile tilapia on natives Cichlidae species in tributary of Amazonas River, Brazil. Biota Amazônia, 4(3), 88-94. https://doi.org/10.18561/2179-5746/biotaamazonia. v4n3p88-94

Cardoso, J. (2008). Um olhar do índio baniwa sobre 'mudança climática': Percepçóes indígenas. En G. Peixoto y S. Andrade (eds.), Rio Negro, Manaus e as mudanças no clima (pp. 15-20). Manaus: Instituto Socioambiental-ISA.

Carey, M. (2010). In the shadow of melting glaciers: Climate change and Andean Society. Nueva York: Oxford University Press. https://doi.org/10.1093/acprof: oso/9780195396065.001.0001

Chocano, L. (2010). Amazonía: pulmón del mundo, hombres y mujeres del Alto Marañón: el pueblo awajún y su percepción del cambio climático. Lima: Fundación PRAIA, Fondo Internacional de Desarrollo Agrícola.

Coordenaçâo das Organizações Indígenas da Amazônia Brasileira-COIAB y Centro Amazônico de Formação Indígena-CAFI (2011). Mudanças climáticas e aquecimento global na visão dos povos indígenas. Manaos: COIAB e CAFI.

Echeverri, J.A. (2009). Pueblos indígenas y cambio climático: el caso de la Amazonía colombiana. Bulletin de l'Institut Français d'Études Andines, 38(1), 13-28. https:// doi.org/10.4000/bifea.2774

Echeverri, J.A. (2010). Percepciones y efectos de cambio climático en grupos indígenas de la Amazonía colombiana. Folia Amazónica, 19(1-2), 85-93. https://doi.org/10.24841/ fa.v19i1-2.344

Edington, J. (2017). Indigenous Environmental Knowledge: Reappraisal. Nueva York: Springer. https://doi.org/10.1007/978-3-319-62491-4

Erfanian, A., Wang, G. y Fomenko, L. (2017). Unprecedented drought over tropical South America in 2016: significantly under-predicted by tropical SST. Scientific Reports, 7(1), 5811. https://doi.org/10.1038/s41598-017-05373-2

Espinosa, O., Gonzáles, G. y Taller de Amazonía (2014). Cambio climático y comunidades indígenas en la Amazonía peruana. En G. Damonte y G. Vila (eds.), Agenda de investigación en temas socioambientales en el Perú: aportes desde las ciencias sociales (pp. 153-192). Lima: Pontificia Universidad Católica del Perú-PUCP.

Espinoza, J.C., Ronchail, J., Guyot, J.L., Junquas, C., Vauchel, P., Lavado, ... y Pombosa, R. (2011a). Climate variability and extreme drought in the upper Solimóes River (Western Amazon Basin): Understanding the exceptional 2010 drought. Geophysical Research Letters, 38, L13406. https://doi.org/10.1029/2011GL047862 
Espinoza, J.C., Ronchail, J., Lavado, W., Santini, W., Vauchel, P., Pombosa, R., ... y Guyot, J.L. (2011b). Las recientes sequías en la cuenca amazónica peruana: orígenes climáticos e impactos hidrológicos. Revista Peruana Geo-Atmosférica (Lima), 3, 63-72.

Espinoza, J.C., Ronchail, J., Guyot, J.L., Junquas, C., Drapeau, G., Martinez, J.M., ... y Espinoza, R. (2012). From drought to flooding: understanding the abrupt 2010-11 hydrological annual cycle in the Amazonas River and tributaries. Environmental Research Letters, 7, 024008. https://doi.org/10.1088/1748-9326/7/2/024008

Espinoza, J.C., Ronchail, J., Frappart, F., Lavado, W., Santini, W. y Guyot, J.L. (2013). The Major Floods in the Amazonas River and Tributaries (Western Amazon Basin) during the 1970-2012 Period: A Focus on the 2012 Flood. Journal of Hydrometeorology, 14(3), 1000-1008. https://doi.org/10.1175/JHM-D-12-0100.1

Espinoza, J.C., Marengo, J.A., Ronchail, J., Molina, J., Noriega, L. y Guyot, J.L. (2014). The extreme 2014 flood in south-western Amazon basin: the role of tropical-subtropical South Atlantic SST gradient. Environmental Research Letters, 9(12), 124007. https://doi.org/10.1088/1748-9326/9/12/124007

Espinoza, J.C., Chavez, S., Ronchail, J., Junquas, C., Takahashi, K. y Lavado, W. (2015). Rainfall hotspots over the southern tropical Andes: Spatial distribution, rainfall intensity, and relations with large-scale atmospheric circulation, Water Resources Research, 51(5), 3459-3475. https://doi.org/10.1002/2014wr016273

Espinoza, J.C., Segura, H., Ronchail, J., Drapeau, G. y Gutierrez-Cori, O. (2016). Evolution of wet-day and dry-day frequency in the western Amazon basin: Relationship with atmospheric circulation and impacts on vegetation. Water Resources Research, 52(11), 8546-8560. https://doi.org/10.1002/2016WR019305

Faulhaber, P. (2004). «As estrelas eram terrenas»: antropologia do clima, da iconografia e das constelaçóes Ticuna. Revista de Antropologia, 47(2), 379-426. https://doi.org/10.1590/ S0034-77012004000200002

Fearnside, P. (2012). La vulnerabilidad de la selva amazónica frente a los cambios climáticos. En II Seminario Internacional sobre la cuenca del río Santiago. Cambio climático. Jalisco: Universidad de Guadalajara. Centro Universitario de Ciencias Económico Administrativas. Recuperado de http://www.cucea.udg.mx/publicaciones/coorinv/ pdf/II_congreso_Rio_Santiag o_Electronico.pdf\#page=15

Fernández-Llamazares, A., Díaz-Reviriego, I., Méndez-López, M.E., Sánchez, I.V., Pyhälä, A. y Reyes-García, V. (2014). Cambio climático y pueblos indígenas: Estudio de caso entre los Tsimane', Amazonía boliviana. Revista Virtual REDESMA, 7(1),110-119.

Ferreira Arza, B. (2011). Saberes locales de colonos e indígenas mosetenes sobre el cambio climático y sus efectos en los modos de vida. La Paz: Soluciones Prácticas-ITDG.

Fundación Bustamante de la Fuente (2010). Cambio climático en el Perú: Amazonía. Lima: Fundación Bustamante de la Fuente. 
Gonzáles, R. (2012). La Chiquitanía y los efectos del cambio climático. Santa Cruz de la Sierra: Programa Ecoclima.

Gullison, R., Frumhoff, P.C., Canadell, J.G., Field, C.B., Nepstad, D.C., Hayoe, K. ... y Nobre, C. (2007). Tropical Forests and Climate Policy. Science, 316(5827), 985-986. https://doi.org/10.1126/science.1136163

Henao, C.I. y Farekatde, G. (2013). Concepción y control del clima entre los hijos del tabaco, la coca y la yuca dulce del resguardo Predio Putumayo, La Chorrera (Amazonas, Colombia). En A. Ulloa y A. Prieto-Rozo (eds.), Culturas, conocimientos, políticas y ciudadanías en torno al cambio climático (pp. 317-349). Bogotá: Universidad Nacional de Colombia, Colciencias.

Hofmeijer, I., Ford, J.D. y Berrang-Ford, L. (2012). Community vulnerability to the health effects of climate change among indigenous populations in the Peruvian Amazon: a case study from Panaillo and Nuevo Progreso. Mitigation and Adaptation Strategies for Global Change. Mitigation and Adaptation Strategies for Global Change, 18(7), 957-978. https://doi.org/10.1007/s11027-012-9402-6

Jiménez-Muñoz, J.C., Mattar, C., Barichivich, J., Santamaría-Artigas, A., Takahashi, K., Malhi, Y., ... y Van der Schrier, G. (2016). Record-breaking warming and extreme drought in the Amazon rainforest during the course of El Niño 2015-2016. Scientific Reports, 6, 33130 https://doi.org/10.1038/srep33130

Kronik, J. y Verner, D. (2010). Indigenous Peoples and Climate Change in Latin America and the Caribbean. Washington D.C.: The World Bank. https://doi.org/10.1596/978-08213-8237-0

Lavado, W., Labat, D., Ronchail, J., Espinoza, J.C. y Guyot, J.L. (2013). Trends in rainfall and temperature in the Peruvian Amazon-Andes basin over the last 40 years (1965-2007). Hydrological Processes, 27(20), 2944-2957. https://doi.org/10.1002/ hyp. 9418

Lewis, S., Brando, P., Phillips, O., van der Heijden, G. y Nepstad, D. (2011). The 2010 Amazon Drought. Science, 331(6017), 554-554. https://doi.org/10.1126/ science. 1200807

Lozano, J. (2016). La actuación femenina frente a los estímulos climáticos en el espacio urbano. Un estudio de caso en la Amazonía peruana (Tesis de Maestría en Estudios Amazónicos). Lima: Pontificia Universidad Católica del Perú, Lima.

Malhi, Y. y Phillips, O. (2004). Tropical forests and global atmospheric change: a synthesis. Philosophical Transactions of the Royal Society of London B, 359(1443), 549-555. https://doi.org/10.1098/rstb.2003.1449

Marengo, J.A., Nobre, C.A., Tomasella, J. y Oyama, M.D., Sampaio de Oliveira, G., Oliveira, R., ... y Brown, I.F. (2008). The drought of Amazonia in 2005. Journal of Climate Change, 21, 495-516. https://doi.org/10.1175/2007JCLI1600.1 
Marengo, J.A., Nobre, C., Tomasella, J., Cardoso, M. y Oyama, M. (2008). Hydro- climatic and ecological behaviour of the drought of Amazonia in 2005. Philosophical Transactions of the Royal Society B: Biological Sciences, 363(1498), 1773-1778. https:// doi.org/10.1098/rstb.2007.0015

Marengo, J.A., Borma, L.S., Rodriguez, D.A., Pinho, P., Soares, W.R. y Alves, L.M. (2013). Recent extremes of drought and flooding in Amazonia: vulnerabilities and human adaptation. American Journal of Climate Change, 2(02), 87-96. https://doi. org/10.4236/ajcc.2013.22009

Marengo, J.A. y Espinoza, J.C. (2015). Extreme seasonal droughts and floods in Amazonia: causes, trends and impacts. International Journal of Climatology, 36(3), 1033-1050. https://doi.org/10.1002/joc.4420

Moutinho, P. y Schwartzman, S. (eds.) (2005). Tropical Deforestation and Climate Change. Belém de Pará: IPAM; Washington DC: Environmental Defense.

Müller, T. (2009). La Luna verde: del medioambiente y su clima. Lima: SPDA.

Novoa, S. (2013). Vulnerabilidade e adaptaçâo sócioecológica diante das mudanças climáticas: caso da comunidade indígena de Gastabala, Ucayali, Perú (Tesis de Maestría). Escola Superior de Conservação e Sustentabilidade (ESCAS), Nazaré Paulista, São Paulo.

Oliveira, V., Mafra, M. y Soares, A. (2012). Eventos climáticos extremos na Amazônia e suas implicaçóes no município de Manaquiri (AM). Revista Geonorte, 1(5), 977-987.

Ortiz, B. (2015). La percepción social del cambio climático. Etnoecología, cambio climático y sabiduría tradicional. Recuperado de http://repositorio.iberopuebla.mx/ handle/20.500.11777/253.

Pabón-Caicedo, J.D., Ycaza, R.P., Friend, F., Espinoza, D., Fenzl, N. y Apostolova, M. (2018). Vulnerabilidad de la cuenca amazónica ante fenómenos hidro climáticos extremos. Cuadernos de Geografia: Revista Colombiana de Geografia, 27(1), 27-49. https://doi.org/10.15446/rcdg.v27n1.56027

Padial, A., Agostinho, A., Azevedo-Santos, V., Frehse, F., Lima-Junior, D., Magalhães, A., ... y Vitule, J. (2017). The «Tilapia Law» encouraging non-native fish threatens Amazonian River basins. Biodiversity and Conservation, 26(1), 243-246. https://doi. org/10.1007/s10531-016-1229-0

Padraza, G. y Pachaguaya, P. (2010). Cuando el sol calienta más. Percepciones del cambio climático de los pueblos indígenas amazónicos de Bolivia, El Puquio-Chiquitano y San José de Uchupiamonas. La Paz: Fundación PRAIA.

Perea, L.M. (2015). Percepciones sobre la variabilidad climática de los pobladores de la comunidad indigena Conta, distrito de Purús, provincia de Purús, departamento de Ucayali (Tesis de Licenciatura en Ingeniería Ambiental). Universidad Nacional del Ucayali, Pucallpa.

Proyecto Andino de Tecnologías Campesinas-PRATEC (2009). Cambio climático y sabiduría andino-amazónica, Perú. Prácticas, percepciones y adaptaciones indígenas. Lima: PRATEC. 
Ramos, C. (2012). Percepçóes sobre mudanças climáticas entre os Baré no Alto Rio Negro (Tesis de Maestría). Universidade Federal de São Carlos, São Carlos, São Paulo.

Remuy, B. (2008). Guardianas de la biodiversidad en la Amazonía colombiana. En A. Ulloa, M. Escobar, L.M. Donato y P. Escobar (eds.), Mujeres indigenas y cambio climático: perspectivas latinoamericanas (pp. 147-150). Bogotá: Universidad Nacional de Colombia, Fundación Natura, UNOCD.

Rosengren, Dan (2016). Science, Knowledge and Belief: On Local Understandings of Weather and Climate Change in Amazonia. Ethnos, 83(4), 607-623. https://doi.org /10.1080/00141844.2016.1213760

Ruiz, M., Müller, T. y Flórez, L. (eds.) (2012a). El clima cambia, cambia tú también. Adaptación al cambio climático en comunidades locales del Perú. Lima: Sociedad Peruana de Derecho Ambiental-SPDA.

Ruiz, M., Müller, T. y Flórez, L. (eds.) (2012b). El clima cambia, cambia tú también. Adaptación al cambio climático en comunidades locales de Colombia. Lima: Sociedad Peruana de Derecho Ambiental-SPDA.

Ruiz, M., Müller, T. y Flórez, L. (eds.) (2012c). El clima cambia, cambia tú también. Adaptación al cambio climático en comunidades locales de Bolivia. Lima: Sociedad Peruana de Derecho Ambiental-SPDA.

Silva, A.L., Cardoso, J.S., Fontes, O., Rodrigues, I.L. (2010). Visóes baniwa sobre mudanças climáticas. En A. Cabalzar, Manejo do mundo: conhecimento e práticas dos povos indígenas do Rio Negro, Noroeste Amazônico (pp. 65-75). Manaos: Federação das Organizaçóes Indígenas do Rio Negro-FOIRN, Instituto Socioambiental-ISA.

Tuesta, E.V. (2014). Percepciones y efectos del cambio climático en grupos indígenas de la Amazonía peruana (Tesis de Licenciatura en Ingeniería agronómica). Universidad Nacional de la Amazonía Peruana, Iquitos.

Ulloa, A. (2008). Implicaciones ambientales y culturales del cambio climático para los pueblos indígenas. En A. Ulloa, M. Escobar, L.M. Donato y P. Escobar (eds.), Mujeres indígenas y cambio climático. Perspectivas latinoamericanas (pp. 17-33). Bogotá: Universidad Nacional de Colombia, Fundación Natura, UNOCD.

United Nations Permanent Forum on Indigenous Issues-UNPFII (2008). Documento de antecedentes. Séptimo período de sesiones (abril 21-mayo 2). El cambio climático, la diversidad biocultural y los medios de vida: la custodia por los pueblos indígenas y nuevos retos. Recuperado de http://www.un.org/esa/socdev/unpfii/documents/ backgrounder\%20climate_ESP_FORMATTED.pdf 